\title{
Direct Measurement of the Gas Entrainment Into a Turbulent Thermal Plasma Jet
}

\author{
Hai-Xing Wang $\cdot$ Fu-Zhi Wei $\cdot$ Xian Meng $\cdot$ Xi Chen • \\ Dong-Sheng Han • Wen Xia Pan
}

Received: 13 September 2010/ Accepted: 2 November 2010/Published online: 17 November 2010

(C) Springer Science+Business Media, LLC 2010

\begin{abstract}
An experimental study is conducted to investigate the entrainment characteristics of a turbulent thermal plasma jet issuing from a DC arc plasma torch operating at atmospheric pressure. The mass flow rate of the ambient gas entrained into the turbulent plasma jet is directly measured by use of the so-called "porous-wall chamber" technique. It is shown that a large amount of ambient gas is entrained into the turbulent plasma jet. With the increase of the gas mass flow rate at the plasma jet inlet or the plasma torch exit, the mass flow rate of entrained ambient gas almost linearly increases but its ratio to the jetinlet mass flow rate decreases. The mass flow rate of the entrained gas increases with the increase of the arc current or jet length. It is also found that using different ways to inject the plasma-forming gas into the plasma torch affects the entrainment rate of the turbulent plasma jet. The entrainment rate expression established previously by Ricou and Spalding (J. Fluid Mech. 11: 21, 1961) for the turbulent isothermal jets has been used to correlate the experimental data of the entrainment rates of the turbulent thermal plasma jet, and the entrainment coefficient in the entrainment rate expression is found to be in range from 0.40 to 0.47 for the turbulent thermal plasma jet under study.
\end{abstract}

Keywords Turbulent plasma jet - Gas entrainment - Entrainment rate measurement . Experimental study

H.-X. Wang $(\bowtie) \cdot$ F.-Z. Wei · D.-S. Han

School of Astronautics, Beijing University of Aeronautics and Astronautics, Beijing 100191, China

e-mail: whx@buaa.edu.cn

X. Meng - W. X. Pan

Institute of Mechanics, Chinese Academy of Sciences, Beijing 100190, China

X. Chen

Department of Engineering Mechanics, Tsinghua University, Beijing 100084, China 


\section{Introduction}

Thermal plasmas jets have been widely used in the field of thermal plasma science and technology, such as thermal plasma spraying, materials processing using a plasma-jet reactor, etc. $[1,2]$. In the plasma spraying or materials processing, ambient gas is often entrained into the plasma jet or the materials processing region, resulting in the spatial evolution of plasma temperature, velocity and species concentration distributions in the plasma jet or the plasma reactor. When the plasma spraying is conducted in atmospheric air surroundings, the entrainment of ambient air into the plasma jet leads to the rapid decrease of plasma temperature and axial velocity and the rapid increase of air or oxygen contents along the axial direction of the plasma jet. The ambient air entrainment would cause the oxidation of the metallic particles injected into the plasma jet and of the metallic substrate material, while the material oxidation is often not desirable for many cases. Although many experimental and modeling results concerning thermal plasma jets have been reported in the literature (see [1-22] and the references cited therein), our understanding about the thermal plasma jet characteristics, especially about the entrainment of ambient gas into the thermal plasma jet, is still incomplete.

Jet entrainment phenomena are also encountered in many other practical situations. For example, gas entrainment controls the flow patterns in combustion chambers, furnaces, chemical reactors, etc. Therefore, the law which governs the gas entrainment into a turbulent jet has been extensively investigated theoretically and experimentally. For an axisymmetrical, isothermal, turbulent gas jet issuing into a similar or dissimilar ambient gas with the same temperature, Ricou and Spalding [23] conducted an experimental study to reveal the relation between the mass flow rate of the entrained gas and that of the turbulent gas jet itself. They used a specially designed porous-wall chamber technique to measure directly the mass flow rate of the ambient gas entrained into the turbulent jet at room temperature. The mass flow rate of the entrained gas was found to increase linearly with increasing axial distance from the jet inlet. From the measurements using a few different gas combinations of the same or different densities (e.g. air into air, propane into air, carbon dioxide into air or hydrogen into air), they established the following entrainment law:

$$
\frac{m}{m_{0}}=C \frac{x}{d_{0}}\left(\frac{\rho_{a}}{\rho_{0}}\right)^{1 / 2}
$$

in which $m$ is the axial mass flux of the turbulent gas jet at the cross section with an axial distance $x$ from the jet inlet; $m_{0}, d_{0}$ and $\rho_{0}$ are the mass flow rate, jet diameter and gas density at the jet inlet; $\rho_{a}$ is the density of entrained ambient gas; whereas $C$ is a dimensionless factor called as entrainment coefficient. It is seen from Eq. 1 that the axial mass flux of the turbulent gas jet is directly proportional to the axial distance $x$ from the jet inlet and the square root of ambient-gas density $\rho_{a}$, but inversely proportional to the jetinlet diameter $d_{0}$ and the square root of jet-inlet gas density. The entrainment coefficient $C$ in Eq. 1 was found to be 0.32 [23] for the isothermal fully-developed turbulent gas jets with dimensionless axial-length $x / d_{0}$ larger than about 13 . Using a short porous-wall chamber movable along the axial direction of the turbulent gas jet, Hill measured the local entrainment rate in the initial region of the isothermal turbulent gas jet with dimensionless length $x / d_{0}$ smaller than 13 [24]. His experimental results showed that the entrainment coefficient $C$ in Eq. 1 increased from about 0.1 at the axial location near the jet inlet to about 0.32 at the location with $x / d_{0}$ about 13 . 
The main difference between the turbulent thermal plasma jet encountered in this study and the turbulent isothermal gas jet studied in [23, 24] is that the plasma jet has much lower jet-inlet gas density and is not isothermal and thus with temperature- or position-dependent gas properties (density, viscosity, etc.). In comparison with the room-temperature jets studied in [23, 24], a flame jet more approximates to the plasma jet. Ref. [23] reported that when the injected gas burned in the jet, the entrainment rate was up to $30 \%$ lower than that when it did not burn. However the strong influence of local heat release and gas expansion in the flame front as it "burned" drastically changes the overall flow structure of the flame jet and makes it difficult to compare the flame jet with the plasma jet directly. Since the plasma jet does not have a localized or concentrated heat source like that in the flame jet, it is actually more analogous to an isothermal jet with low jet-inlet gas density and high ambient-gas density (e.g. for the gas combination $\mathrm{H}_{2}$ into air if the hydrogen does not burn in the air). Hence, the entrainment characteristics of the turbulent plasma jet may still be referenced from the research results of isothermal turbulent gas jets.

The gas entrainment into a turbulent thermal plasma jet was involved in many earlier studies [1-25]. A variety of diagnostic techniques have been employed to study the entrainment of ambient gas into the turbulent plasma jet, and many measured results have also been reported concerning the evolution of plasma parameters in plasma jets $[5,6,9$, 10, 14-16]. The modeling results reported in [19-21] showed that since turbulent transport mechanism is dominant in the turbulent plasma jet, the mass flow rate of the ambient air entrained into the turbulent plasma jet is comparatively large (e.g. maybe about 20 times larger than the mass flow rate at the jet inlet for a turbulent thermal plasma jet with 100$\mathrm{mm}$ length) and almost directly proportional to the mass flow rate at the jet inlet. However, those predicted results about the entrainment characteristics of the turbulent thermal plasma jet have not been checked experimentally. The entrainment characteristics of cold turbulent gas jets have been well studied experimentally, but so far corresponding experimental results under thermal plasma conditions are not available in the literature. This study aims to present some experimental results concerning the entrainment characteristics of the turbulent thermal plasma jet.

\section{Experimental Method}

The experimental method used in this study is similar to that employed by Ricou and Spalding [23], i.e. the so-called porous-wall technique is used to measure directly the entrainment rate of turbulent plasma jet. As shown in Fig. 1 for the experimental setup, a cylindrical chamber with a small downstream opening is installed co-axially with the plasma torch. The cylindrical chamber encloses the upstream region of the turbulent plasma jet in order to control the flow rate of the gas being entrained into this upstream region of the plasma jet. Inside the cylindrical chamber there is a coaxial porous wall made up of fine-hole filter cloth winded on a framework, through which the gas supplied for jet entrainment can flow in a radially inward direction. If the turbulent plasma jet is a completely free one (without the existence of the cylindrical chamber), ambient gas will be freely entrained into the jet and the whole jet will be with almost uniform pressure. On the other hand, if the turbulent plasma jet is partially enclosed by the porous-wall chamber with a small downstream opening (as in Fig. 1) and gas is supplied through the porous-wall and flowing inward into the jet region to act as the entrained gas of the turbulent plasma jet, a pressure difference $\Delta P$ will appear generally between the porous-wall chamber interior and the atmospheric air surroundings outside the cylindrical chamber, depending on the 


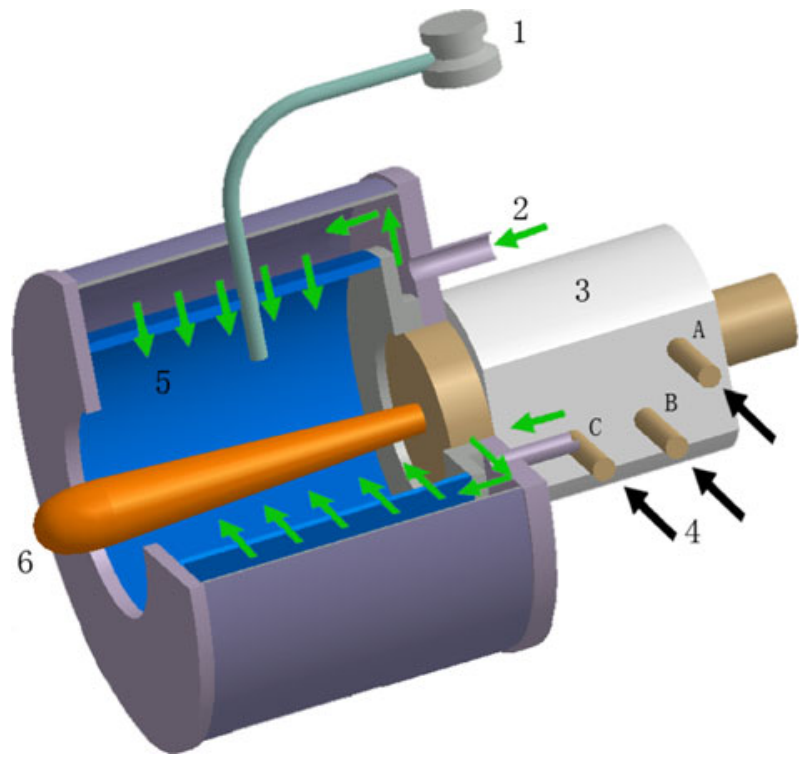

Fig. 1 Schematic diagram of the experimental setup. 1 pressure sensor, 2 entrained gas, 3 plasma torch, 4 plasma-forming gas, 5- porous wall, 6 plasma jet, $A$ gas inlet $A, B$ gas inlet $B, C$ gas inlet $C$

mass flow rate of the supplied gas. If the supplied gas flow rate is larger than that could be entrained by the plasma jet, $\Delta P$ will be $>0$, while $\Delta P<0$ will appear when the supplied gas flow rate is less than that required by the jet entrainment [23, 24]. Hence, the measured mass flow rate of the gas supplied through the porous-wall when $\Delta P=0$ will be equal to the jet entrainment rate. In this experiment, the pressure difference $\Delta P$ is very small, which is typically of the order of several $\mathrm{Pa}$ for the turbulent plasma jet. So a sensitive pressure sensor has been used in the experiment to measure the pressure difference.

The plasma torch used to generate the turbulent plasma jet is a DC non-transferred arc plasma torch, which consists of a $\mathrm{Ce}-\mathrm{W}$ cathode, an insulating inter-electrode insert and an anode-nozzle, as shown in Fig. 2. The exit diameter of the anode-nozzle of the plasma torch is $8 \mathrm{~mm}$ and the plasma-forming gas is argon. The argon is admitted into the plasma torch in three ways, i.e. axially from the annular slot A at the cathode upstream end, tangentially from the hole $\mathrm{B}$ around the upstream end of the cathode and also tangentially from the hole $\mathrm{C}$ near the upstream section of the anode (see Fig. 2). $\mathrm{N}_{2}$ is employed as the entrained gas in this experiment. The argon mass flow rate of the plasma torch is measured by an electromagnetic flow-meter with a full-scale accuracy of $1.5 \%$, while the mass flow rate of the entrained gas (nitrogen) was measured by a rotameter with a full-scale accuracy of $2.5 \%$. Electric insulation is ensured between the plasma torch and the cylindrical chamber. In a preliminary experiment, it is found that the flow-rate ratios of the gases through the three different ways have some influence on the entrainment rate of the turbulent plasma jet even for the case with the same total flow rate of argon. So in this experiment, besides studying the effects of the working parameters of the plasma torch (total argon flow rate and arc current) on the jet entrainment, we also examine the effects of the gas mass flow rate supplied through each of the three different ways on the entrainment characteristics of the turbulent plasma jet. 


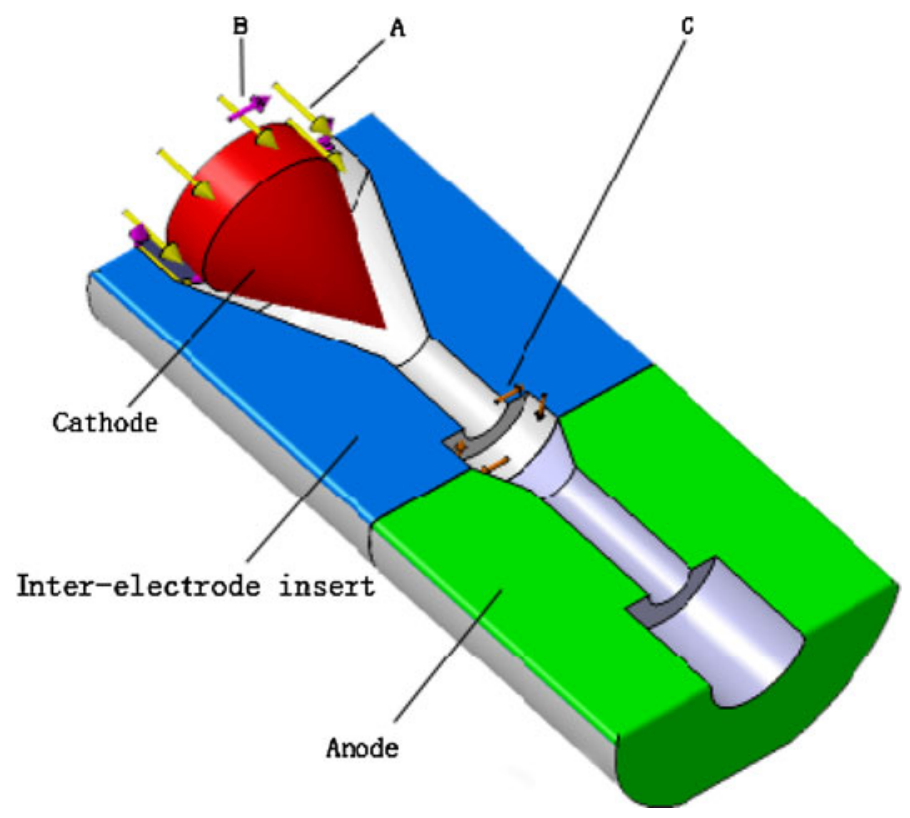

Fig. 2 Schematic diagram of the plasma torch used in this experiment

\section{Results and Discussion}

The size of the aperture at the downstream end of the cylindrical porous-wall chamber has to be chosen carefully. If the opening diameter at the downstream end of the cylindrical porous-wall chamber or the opening angle $\theta$ defined by

$$
\theta=2 \tan ^{-1}\left(0.5 D_{\mathrm{ex}} / L_{\mathrm{ch}}\right)
$$

is too small, the downstream-end wall of the cylindrical chamber will interfere with the axial flow of the plasma jet itself while such an effect should be avoided. $L_{\mathrm{ch}}$ in Eq. 2 is the length of the porous-wall chamber, whereas $D_{\mathrm{ex}}$ is the opening diameter. On the other hand, in order to obtain an easily detected pressure difference, the opening diameter or the opening angle $\theta$ should be reasonably small. Figure 3 shows the measured results leading to our choice of the opening angle $\theta$ or the opening diameter $D_{\text {ex }}$. For the case with argon mass flow rate $m_{0}=0.239 \mathrm{~g} / \mathrm{s}\left(m_{0}=m_{\mathrm{A}}+m_{\mathrm{B}}+m_{\mathrm{C}}, m_{\mathrm{A}}=0.104 \mathrm{~g} / \mathrm{s}, m_{\mathrm{B}}=0.080 \mathrm{~g} / \mathrm{s}, m_{\mathrm{C}}=\right.$ $0.055 \mathrm{~g} / \mathrm{s}$ ) and arc current $I=140 \mathrm{~A}$ of the plasma torch and porous-wall chamber length $L_{\mathrm{ch}}=110 \mathrm{~mm}$, the measured variations of pressure difference $\Delta P$ with the flow rate ratio $m_{1} / m_{0}$ are shown in Fig. 3 for three different opening angles, i.e. $\theta=25.6^{\circ}, 30.5^{\circ}$ and $35.3^{\circ}$ (corresponding to the opening diameters of 50,60 and $70 \mathrm{~mm}$ ), respectively. $\Delta P$ presented in Fig. 3 is the measured pressure difference between the porous-wall chamber interior and the atmospheric air surroundings outside the porous-wall chamber, whereas $m_{1} / m_{0}$ is the ratio of the mass flow rate of nitrogen inflowing inward through the porous wall, $m_{1}$, to the mass flow rate of the plasma-forming gas (argon) of plasma torch, $m_{0}$. At smaller $\theta$ angle (i.e. $25.6^{\circ}$ or $30.5^{\circ}$ ), steeper variation of the pressure difference with $m_{1} / m_{0}$ is observed. Almost the same values of the mass flow-rate ratio, i.e. $m_{1} / m_{0}=25.5$, are obtained as $\Delta P=$ 0 . On the other hand, when $\theta=35.3^{\circ}$ is used, the measuring accuracy appreciably 


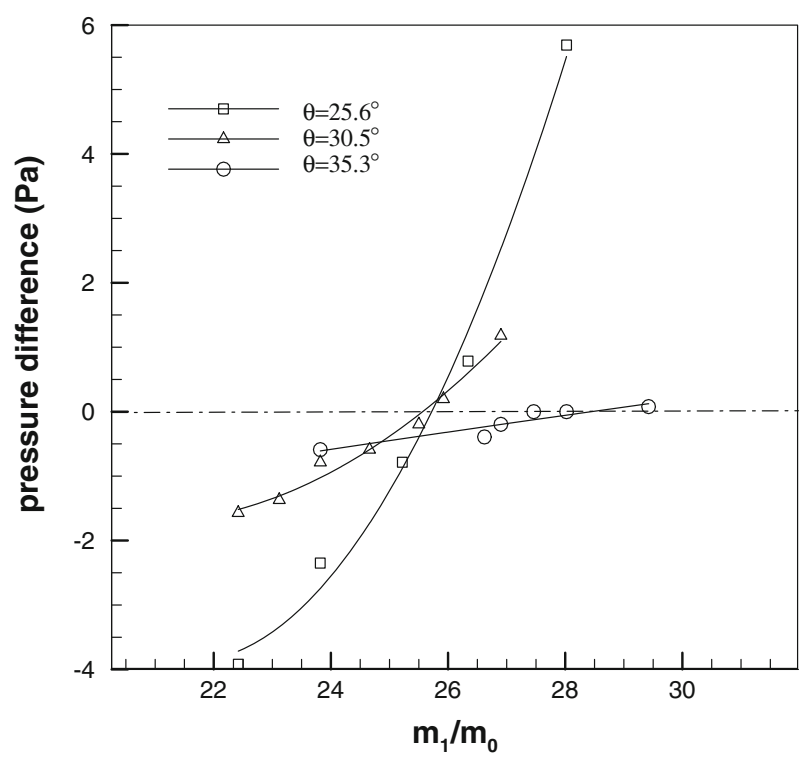

Fig. 3 Variation of measured pressure difference $\Delta P$ with the mass flow rate ratio $m_{1} / m_{0}$ for three different opening angles

decreases. These tests lead to our choice of $\theta=30.5^{\circ}\left(D_{\mathrm{ex}}=60 \mathrm{~mm}\right)$ in all the subsequent experiments, and the mass flow rate of entrained gas $m_{1}$ measured as $\Delta P=0$ will be called the entrained gas mass flow rate or the entrainment rate of the turbulent plasma jet. In the following, $m_{1}$ always expresses the entrained gas mass flow rate.

The measured variations of entrained gas mass flow rate, $m_{1}$, with the jet-inlet flow rate, $m_{0}$, are shown in Fig. 4 for three different arc currents of the plasma torch (i.e. $I=140,160$ and $180 \mathrm{~A}$, respectively) but with a fixed porous-wall chamber length $L_{\mathrm{ch}}=90 \mathrm{~mm}$. It is seen that the entrainment rate or the mass flow rate of nitrogen entrained into the turbulent argon plasma jet, $m_{1}$, increases almost linearly with increasing jet-inlet mass flow rate, $m_{0}$. Fig. 4 also indicates that the larger the arc current, the larger is the gas mass flow-rate entrained into the turbulent plasma jet. The experimental results presented in Fig. 4 are replotted in Fig. 5 where the variations of the entrained gas mass flow rate normalized to the jet-inlet mass flow rate $\left(m_{1} / m_{0}\right)$ are shown as the function of the jet-inlet mass flow rate $\left(m_{0}\right)$ for the three different arc currents. It is seen from the Fig. 5 that the values of the mass flow rate ratio $m_{1} / m_{0}$ are in the range of 20-28 and that ratio $m_{1} / m_{0}$ decreases with increasing jet-inlet mass flow rate.

Effects of the porous-wall chamber length, $L_{\mathrm{ch}}$, on the mass flow rate of nitrogen entrained into the turbulent argon plasma jet are shown in Fig. 6 for the case with a fixed arc current of plasma torch $(I=160 \mathrm{~A})$. Figure 6 also demonstrates that the mass flow rate of nitrogen entrained into the turbulent argon plasma jet increases approximately linearly with the jet-inlet mass flow-rate, and that the mass flow rate of nitrogen entrained into the turbulent argon plasma jet increases with increasing porous-wall chamber length, as expected. The experimental results presented in Fig. 6 are re-plotted in Fig. 7, where the variations of the entrained mass flow rate normalized to the jet-inlet mass flow rate $\left(m_{1} / m_{0}\right)$ are shown as the function of the jet-inlet mass flow rate $\left(m_{0}\right)$ for the three different chamber lengths. It is seen that the value of the normalized mass flow rate $m_{1} / m_{0}$ increases with 
Fig. 4 Variation of the entrainment rate $m_{1}$ with the jetdifferent arc currents of the plasma torch $\left(L_{\mathrm{ch}}=90 \mathrm{~mm}\right)$ inlet mass flow rate $m_{0}$ for

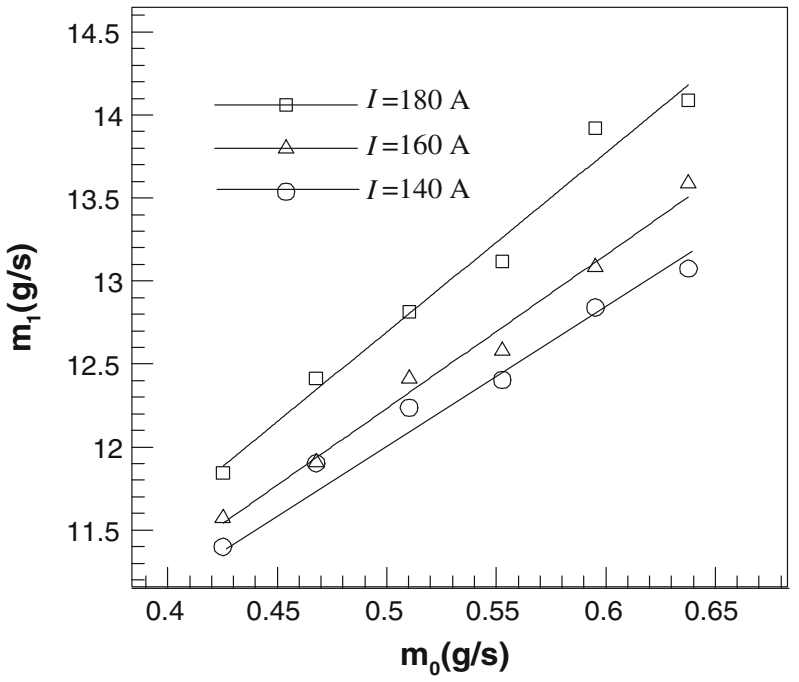

Fig. 5 Variation of the mass flow rate ratio $m_{1} / m_{0}$ with the jetinlet mass flow rate $m_{0}$ for different arc currents of the plasma torch $\left(L_{\mathrm{ch}}=90 \mathrm{~mm}\right)$

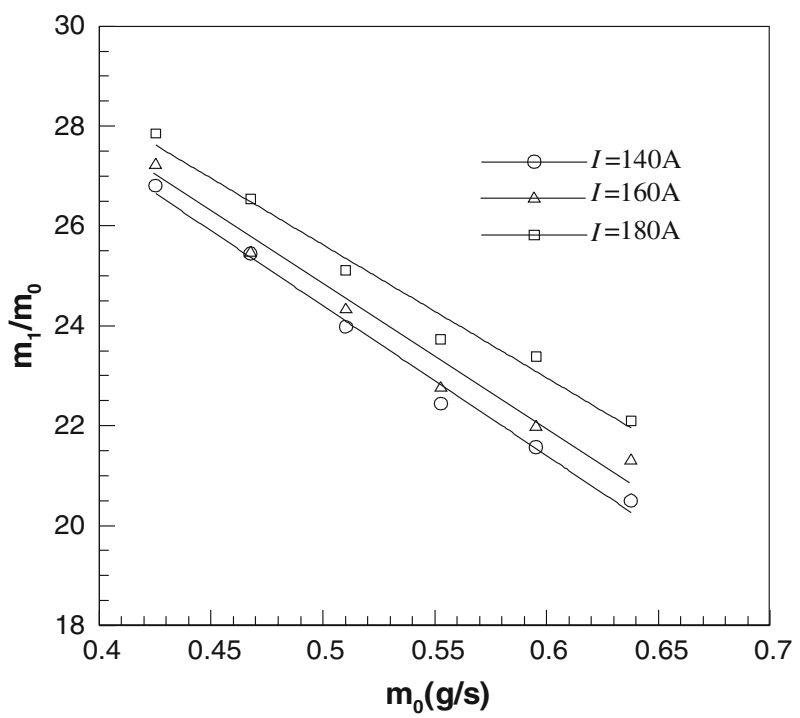

increasing porous-wall chamber length $\left(L_{\mathrm{ch}}\right)$ but decreases with increasing jet-inlet mass flow rate $\left(m_{0}\right)$. The values of the mass flow rate ratio $m_{1} / m_{0}$ are in the range of 21-29.

The modeling study on the entrainment characteristics of turbulent argon thermal plasma jets issuing into ambient air was carried out in [19-21]. It was shown that the entrained air mass flow-rate almost linearly increases with increasing jet-inlet gas velocity (or mass flow rate) for a fixed jet-inlet temperature and with increasing axial distance. And the values of the entrained gas mass flow rate normalized to the jet-inlet mass flow rate 


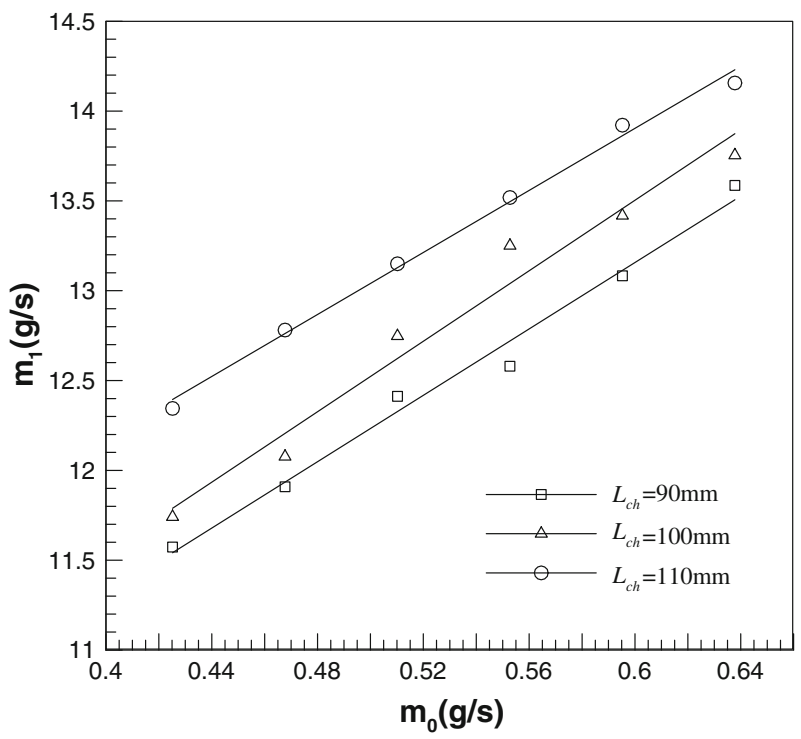

Fig. 6 Variation of the entrainment rate $m_{1}$ with the jet-inlet flow rate $m_{0}$ for different porous-wall chamber lengths $(I=160$ A)

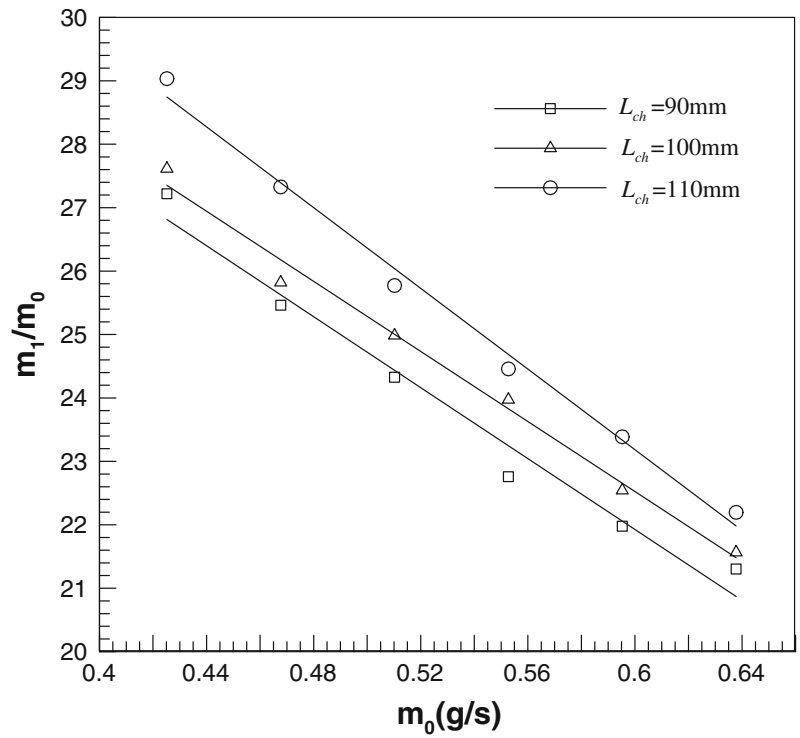

Fig. 7 Variation of the mass flow rate ratio $m_{1} / m_{0}$ with the jet-inlet flow rate $m_{0}$ for different porous-wall chamber lengths $(I=160 \mathrm{~A})$

$\left(m_{1} / m_{0}\right)$ were as large as about 16 at the axial distance of $100 \mathrm{~mm}$ [19]. The present experimental results presented in Figs. 4-7 are roughly consistent with the modeling predictions of Ref. [19-21], although the entrained gases are somewhat different (nitrogen is used in this experiment while air is used in the modeling studies). 
As mentioned above, the plasma-forming gas (argon) of the plasma torch is supplied through three channels, i.e. through the inlet A (inflowing axially from the upstream annular slot of plasma torch), the inlet B (inflowing tangentially near the upstream end of plasma torch) and the inlet $\mathrm{C}$ (inflowing tangentially near the upstream section of anodenozzle), respectively. In the experiment, it is found that changing the gas mass flow rate inflowing into the plasma torch from the inlet $\mathrm{A}, \mathrm{B}$ or $\mathrm{C}$ has different effects on the entrainment rate of the turbulent plasma jet. Fig. 8 shows the variations of the measured entrainment rate with the jet-inlet mass flow rate for the case with fixed arc current $I=150$ A and chamber length $L_{\mathrm{ch}}=90 \mathrm{~mm}$, while the total argon mass flow rate is changed by changing only the mass flow rate through the inlet A, B, or C. Namely, curve A in Fig. 8 represents the Case $\mathrm{A}$ in which the total argon mass flow rate $m_{0}$ is changed only by changing the gas mass flow rate through the inlet $\mathrm{A}$, while the mass flow rates of argon inflowing from the inlets $\mathrm{B}$ and $\mathrm{C}$ are fixed, i.e. $m_{\mathrm{B}}=0.085 \mathrm{~g} / \mathrm{s}$ and $m_{\mathrm{C}}=0.085 \mathrm{~g} / \mathrm{s}$. Similarly, the curve B represents the Case B in which $m_{0}$ is changed only by changing $m_{\mathrm{B}}$, while the other two flow rates are fixed, i.e. $m_{\mathrm{A}}=0.085 \mathrm{~g} / \mathrm{s}$ and $m_{\mathrm{C}}=0.085 \mathrm{~g} / \mathrm{s}$. The curve $\mathrm{C}$ represents the Case $\mathrm{C}$ in which $m_{0}$ is changed only by changing $m_{\mathrm{C}}$, while the other two flow rates are fixed, i.e. $m_{\mathrm{A}}=0.085 \mathrm{~g} / \mathrm{s}$ and $m_{\mathrm{B}}=0.085 \mathrm{~g} / \mathrm{s}$. The corresponding variations of the normalized mass flow rate $\left(m_{1} / m_{0}\right)$ with the jet-inlet mass flow rate are shown in Fig. 9 for the Cases A, B and C. It is seen from Figs. 8 and 9 that the mass flow rate $m_{\mathrm{A}}$ inflowing into the plasma torch through the annular inlet A, i.e. axially inflowing gas, has more significant influence on the entrainment rate of turbulent plasma jet. The mass flow rate $m_{\mathrm{C}}$ tangentially inflowing into the plasma torch from the inlet $\mathrm{C}$ less affects the plasma jet entrainment compared with that from the inlet $\mathrm{A}$ or the inlet $\mathrm{B}$. Those experimental results imply that swirling of the turbulent plasma jet would lead to the decrease the entrainment rate of the turbulent jets. The stronger the swirling (e.g. the gas is tangentially injected from the downstream inlet $\mathrm{C}$ instead from inlet $\mathrm{B}$ ), the more the entrainment rate decreases.

Although it is anticipated that Eq. 1 obtained in $[23,24]$ for the turbulent isothermal gas jet is not completely applicable to the highly non-isothermal thermal plasma jet under

Fig. 8 Variation of the entrainment rate $m_{1}$ with the jetinlet mass flow rate $m_{0} . m_{0}$ is changed by changing the flow rate inflowing from inlet $\mathrm{A}, \mathrm{B}$ or $\mathrm{C}\left(I=150 \mathrm{~A}, L_{\mathrm{ch}}=90 \mathrm{~mm}\right)$

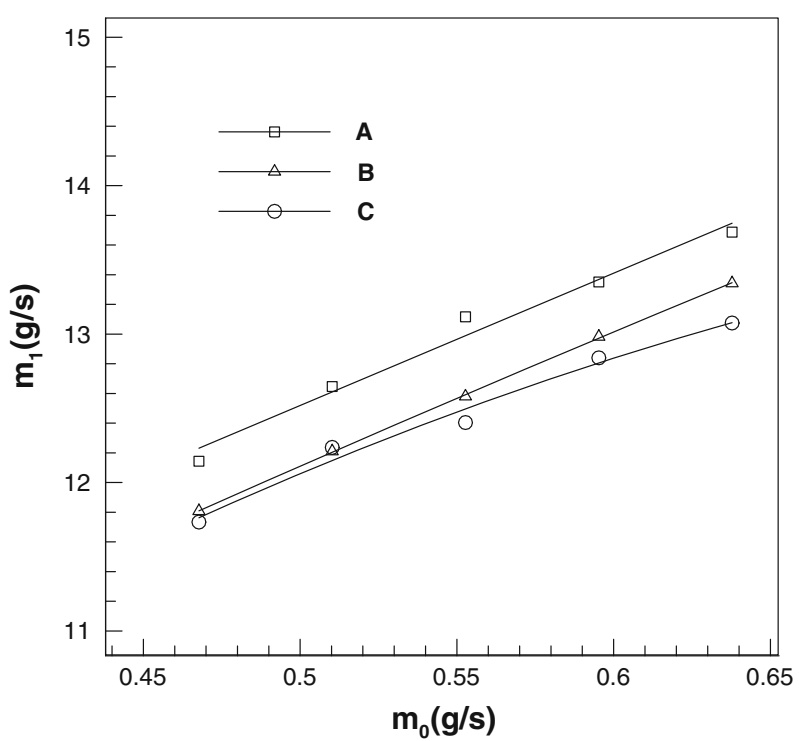


Fig. 9 Variation of the mass flow rate ratio $m_{1} / m_{0}$ with the jetinlet mass flow rate $m_{0} . m_{0}$ is changed by changing the flow rate inflowing from inlet $\mathrm{A}, \mathrm{B}$ or $\mathrm{C}\left(I=150 \mathrm{~A}, L_{\mathrm{ch}}=90 \mathrm{~mm}\right)$

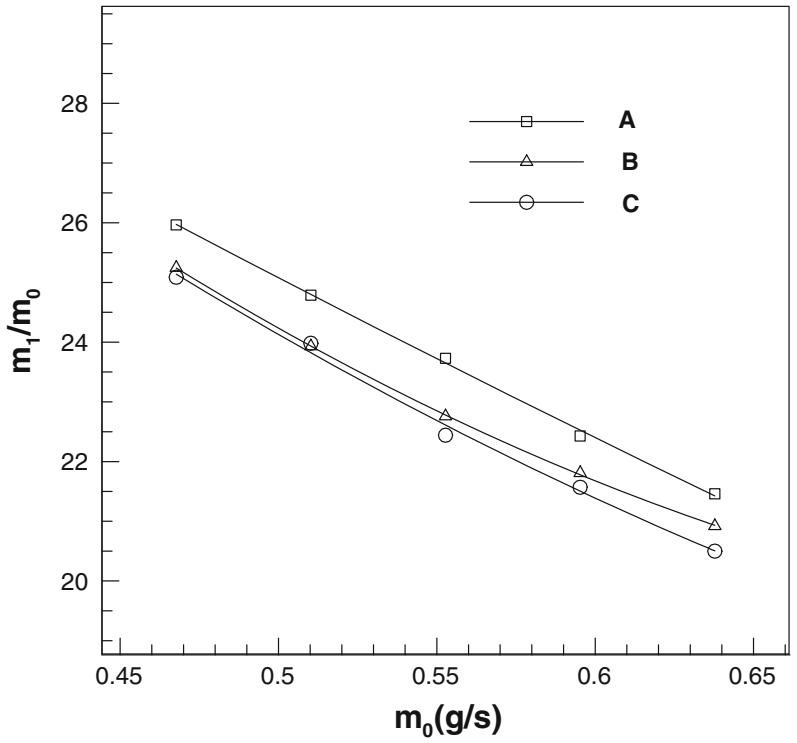

study, Eq. 1 is still useful for understanding the entrainment of ambient gas into the thermal plasma jet. As is seen above, the experimental results obtained under the present thermal plasma conditions also show that the entrained mass flow rate almost linearly increases with the increase of the jet-inlet mass flow rate $m_{0}$ and of the porous-wall length $L_{\mathrm{ch}}$. Hence, it is worth examining whether Eq. 1 can be used to estimate roughly the mass flow rate of the gas entrained into the turbulent thermal plasma jet.

In the present study, the dimensionless lengths $x / d_{0}$ are $11.25,12.50$ and 13.75 , respectively, the entrained gas density $\rho_{a}$ is known for nitrogen at room temperature, whereas the values of the axial mass flux $m$ or the mass flow rate ratio $m / m_{0}$ can be calculated according the measured data $\left(m=m_{0}+m_{1}\right)$. Hence, as long as the effective gas density $\rho_{O}$ at the plasma jet inlet is determined, the entrainment coefficient $C$ in Eq. 1 could be evaluated. Because the temperature profile at the plasma torch exit or jet inlet is nonuniform, the average gas temperature at the plasma torch exit is used to estimate the effective density $\rho_{0}$. The average gas temperature at the plasma torch exit can be calculated using the following energy balance relation:

$$
m_{0} \int_{T_{0}}^{\bar{T}} c_{p} d T=\eta U I
$$

where $m_{0}$ is the argon mass flow rate at the plasma torch exit; $T_{0}$ is the room temperature $\left(T_{0}=300 \mathrm{~K}\right) ; c_{p}$ is temperature-dependent argon specific heat at constant pressure; $\bar{T}$ is the average temperature at the plasma jet inlet; $\eta$ is the thermal efficiency of the plasma torch; whereas $U$ and $I$ are the measured arc voltage and arc current. As an example, for the case with $m_{0}=0.638 \mathrm{~g} / \mathrm{s}$ and $I=140 \mathrm{~A}$, the measured arc voltage is $U=50 \mathrm{~V}$. Pan et al. [25] measured the thermal efficiency of the same plasma torch as used in this study, the measured value was about 0.40 for the turbulent argon plasma jet. If we use the measured thermal efficiency $\eta=0.40$ in Eq 3, the average jet-inlet temperature $\bar{T}$ would be $8470 \mathrm{~K}$, corresponding to effective argon density $\rho_{0}=5.73 \times 10^{-2} \mathrm{~kg} / \mathrm{m}^{3}$. Similar estimations of 
Fig. 10 Correlation of the entrainment rates for the turbulent thermal plasma jet

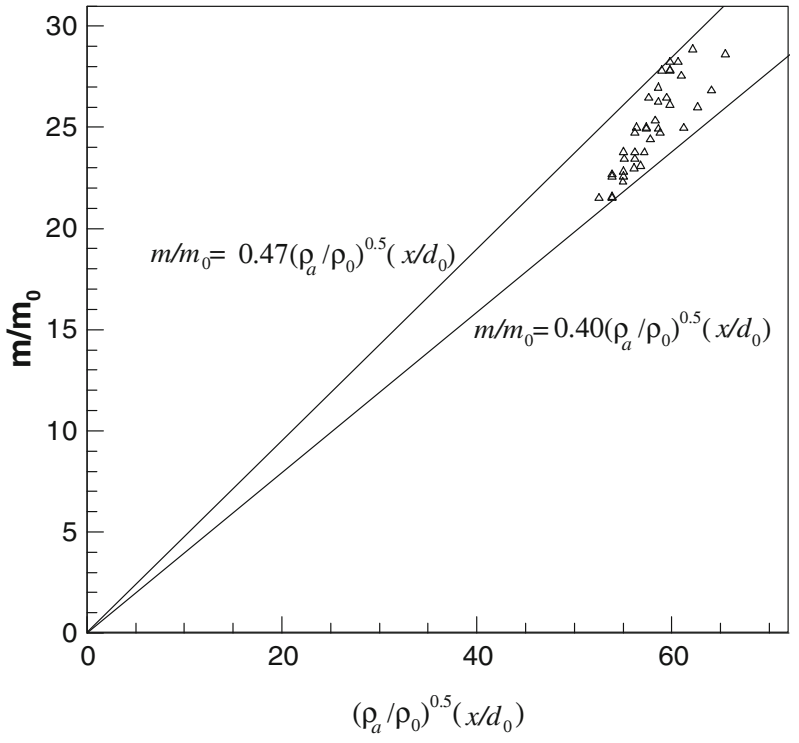

the average jet-inlet temperature $\bar{T}$ and corresponding effective density $\rho_{0}$ are also performed for other combinations of $m_{0}, I$ and $U$.

Now we can use Eq. 1 to correlate our experimental data to estimate the entrainment coefficient $C$ for the turbulent thermal plasma jet. All the processed experimental data have been presented in Fig. 10 in the dimensionless form. Figure 10 shows that all the measured entrainment rate data are distributed in the area between the line expressing $m / m_{0}=$ $0.40\left(\rho_{a} / \rho_{0}\right)^{1 / 2}\left(x / d_{0}\right)$ and the line expressing $m / m_{0}=0.47\left(\rho_{a} / \rho_{0}\right)^{1 / 2}\left(x / d_{0}\right)$. This result means that for the studied case the entrainment coefficient $C$ for turbulent thermal plasma jets are in the range $0.40-0.47$, or $\mathrm{C}=0.435 \pm 0.035$, which are somewhat larger than the entrainment coefficient 0.32 obtained for the isothermal gas jets [23, 24]. It is believed to be due to the highly non-isothermal feature of the turbulent thermal plasma jet.

\section{Conclusions}

The "porous-wall chamber" technique has been used to measure directly the entrainment rate of a turbulent thermal plasma jet. It is found that the mass flow-rate of nitrogen entrained into the turbulent argon plasma jet almost linearly increases with increasing mass flow rate at the jet inlet. The entrained gas mass flow-rate increases with the increase of the porous-wall chamber length and the arc current of the plasma torch. The measured results show that using different ways to inject the working gas into the plasma torch somewhat affects the entrainment rate of the turbulent plasma jet, and that the swirling of the turbulent plasma jet would lead to the decrease the entrainment rate of the turbulent plasma jet. Eq. 1 can be used to roughly evaluate the entrainment rate of the turbulent thermal plasma jet with the estimated values of the entrainment coefficient in the range of $0.40-0.47$ for the studied case.

Acknowledgment This work was supported by the National Natural Science Foundation of China (Grant No. 10772016). 


\section{References}

1. Pfender E (1999) Thermal plasma technology: where do we stand and where are we going? Plasma Chem Plasma Process 19(1):1-31

2. Fauchais P (2004) Understanding plasma spraying. J Phys D Appl Phys 37:R86-108

3. El-Kaddah N, McKelliget J, Szekely J (1984) Heat transfer and fluid flow in plasma spraying. Metallurg Trans B 15B:59-70

4. Chyou YP, Pfender E (1989) Modeling of plasma jets with superimposed vortex flow. Plasma Chem Plasma Process 9:291-328

5. Capetti A, Pfender E (1989) Probe measurements in argon plasma jets operated in ambient argon. Plasma Chem Plasma Process 9:329-341

6. Pfender E, Fincke J, Spores E (1991) Entrainment of cold gas into thermal plasma jets. Plasma Chem Plasma Process 11:529-543

7. Chang CH, Ramshaw JD (1993) Numerical simulations of argon plasma jets flowing into cold air. Plasma Chem Plasma Process 13:189-209

8. Pfender E (1994) Plasma jet behavior and modeling associated with the plasma spray process. Thin Solid Films 238:228-241

9. Fincke JR, Chang CH, Swank WD, Haggard DC (1994) Entrainment and demixing in subsonic thermal plasma jets: comparison of measurements and predictions. Int J Heat Mass Transfer 37:1673-1682

10. Jankovic M, Mostaghimi J (1995) A new nozzle design for dc plasma spray guns. Plasma Chem Plasma Process 15:607-628

11. Huang PC, Heberlein J, Pfender E (1995) A two-fluid model of turbulence for a thermal plasma jet. Plasma Chem Plasma Process 15:25-46

12. Kang KD, Hong SH (1999) Numerical analysis of shroud gas effects on air entrainment into thermal plasma jet in ambient atmosphere of normal pressure. J Appl Phys 85:6373-6381

13. Li HP, Chen X (2002) Three-dimensional modeling of the turbulent plasma jet impinging upon a flat plate and with transverse particle and carrier-gas injection. Plasma Chem Plasma Process 22:27-58

14. Fincke JR, Crawford DM, Snyder SC, Swank WD, Haggard DC, Williamson RL (2003) Entrainment in high-velocity, high-temperature plasma jets. Part I: experimental results. Int J Heat Mass Transfer 46:4201-4213

15. Williamson RL, Fincke JR, Crawford DM, Snyder SC, Swank WD, Haggard DC (2003) Entrainment in high-velocity, high-temperature plasma jets. Part II: computational results and comparison to experiment. Int J Heat Mass Transfer 46:4215-4228

16. Choi SS, Hwang TH, Seo JH, Kim DU, Hong SH (2004) Effects of anode nozzle geometry on ambient air entrainment into thermal plasma jets generated by nontransferred plasma torch. IEEE Trans Plasma Sci 32:473-478

17. Cheng K, Chen X (2004) Prediction of the entrainment of ambient air into a turbulent argon plasma jet using a turbulence-enhanced combined-diffusion-coefficient method. Int $\mathrm{J}$ Heat Mass Transfer 47:5139-5148

18. Cheng K, Chen X, Wang HX, Pan WX (2006) Modeling study of shrouding gas effects on a laminar argon plasma jet impinging upon a flat substrate in air surroundings. Thin Solid Films 506-507:724-728

19. Cheng K, Chen X, Pan WX (2006) Comparison of laminar and turbulent thermal plasma jet characteristics-a modeling study. Plasma Chem Plasma Process 26:211-235

20. Wang H-X, Chen X, Pan WX (2007) Modeling study on the entrainment of ambient air into subsonic laminar and turbulent argon plasma jets. Plasma Chem Plasma Process 27:141-162

21. Wang H-X, Chen X, Pan WX (2008) Effects of the length of a cylindrical solid shield on the entrainment of ambient air into turbulent and laminar impinging argon plasma jets. Plasma Chem Plasma Process 28:85-105

22. Chen X (2009) Heat transfer and fluid flow under thermal plasma conditions. Science Press, Beijing (in Chinese)

23. Ricou FP, Spalding DB (1961) Measurements of entrainment by axisymmetrical turbulent jets. J Fluid Mech 11:21-32

24. Hill HJ (1972) Measurement of local entrainment rate in the initial region of axisymmetric turbulent air jets. J Fluid Mech 51:773-779

25. Pan WX, Zhang WH, Ma W, Wu CK (2002) Characteristics of argon laminar dc plasma jet at atmospheric pressure. Plasma Chem Plasma Process 22:271-283 